\title{
Zweiter Beitrag zur Coleopteren-Fauna des Kankasus.
}

\author{
Von Eug. Koenig in Tiflis. ${ }^{\mathbf{1}}$ )
}

Tetracha euphratica Dej. var. armeniaca Dokht. war bisher aus dem Kaukasus nur von Saljan (Kuramündung) bekannt. Am 13. Juni 1894 fand ich ein Exemplar am Salzsee Temirgoje, zirka 30 Kilometer westlich von Petrowsk und erbeutete zwei Stücke dieser Art im Kreise Aresch, Gour. Elisabethpol am 1. Juni 1902. Diese schöne Art dürfte wohl noch weiter nordwestlich von Getrowsk zu finden sein, wahrscheinlich wird sie auch in der Kura-Niederung weiter hinauf reichen. Dr. O. Schneider bemerkt in »Die Käferfauna von Ramleh bei Alexandrien (Isis, 1571, Heft I. Sep. p. 2), daß diese Art flügellos sei. Das ist aber ein Irrtum, der hiermit widerlegt sein mag. Sie hat vollständig ausgebildete Flügel, die sie sehr gut zu benützen versteht, wie ich mich in Transkaspien und im Kreise Aresch überzeugen konnte, wo sie ans Licht geflogen aber nicht gelaufen - kam.

\section{Tribax Bibersteini Mén. var. nov. Maljushenkoi m.}

Ganglbauer, D. E. Z. 1886. 330 und Reitter, Bestimm.-Tab. XXXIV,p. 109 beziehen den Tr. Puschlimi Kol. auf Tr. Kolenatii Chaud. Leider kann ich die Originalbeschreibung von Chaudoir nicht einsehen. Nach Ganglbauer und Reitter sind bei letzterer drt das erste Fühlerglied und die Schenkel schwarz. Nun sagt aber Kolenati (Meletem. Entom. Fasc. I. 1845, p. 27) in der Beschreibung ausdrücklich . . . . antennarum articulus primus ruber . . . . Crura rubra incrassata basi apiceque nigra .... und weiter in der Anmerkung (1. e. p. 28) Carabus Biebersteinii Mén ..... species constans, proxima quidem nostro, antennis autem et pedibus nigris sat distincta. Kolenati beschreibt nun seinen Platychrus Puschlimi von zwei verschiedenen Fundorten, vom Kasbek und vom Sarijal, Gouv. Elisabethpol. Nach meinen Untersuchungen sind es zwei, wenn auch sich sehr nahestehende, jedoch verschiedene Lokalrassen. Die Stücke vom Kasbek und im Zentralkaukasus bilden die var. Kasbekianus Kr., die vom Sarijal stammenden nenne ich var. Maljushenkioi. Letztere unterscheidet sich von Kasbeliamus durch 1) ef. W. E. Z. 1901. p. 9. 
relativ stärker erhabene Zwischenräume und Kettenstreifen, besonders aber durch folgende zwei Merkmale, die sich an einer Reihe von Exemplaren als konstant erwiesen haben. Bei var. Maljushenkioi ist der hintere Supraorbitalpunkt doppelt so weit vom Auge entfernt als bei var. Kasbeliamus, sodann finde ich bei ersterer Art am Innenrande der Hinterschenkel $1-2$ borstentragende Punkte (nur in einem Falle fand ich auf einer Seite 3), während bei letzterer 4-5 Punkte vorhanden sind. 'Ich erhielt eine Anzahl dieser Form von Dr. Maljushenko, welcher sie auf den Ausläufern des Sarijal im Gouv. Elisabethpol sammelte.

Scarites (Parallelomorphus) salimus Dej. In den Bestimmungstabellen XXXIX. wird diese Art nur durch das Vorhandensein eines Porenpunktes auf dem dritten Zwischenraume der Flügeldecken von Sc. eurytus Fisch. und cylindronotus Fald. getrennt. Unter meinen zahlreichen Stücken fanden sich aber 2 Exemplare, bei denen der Porenpunkt einseitig ist, bei einem fehlten jedoch die Punkte ganz. Da nun solche Exemplare ohne Porenpunkte nach obiger Tabelle nicht sicher zu bestimmen sind, so gebe ich hier eine neue Uebersicht das Subg. Parallelomorphus:

1" Seiten des Halsschildes ohne zähnchenartig vortretende Vorderwinkel. Dritter Zwischenraum der Flügeldecken (mit Ausnahme des Borstenpunktes am Ende des zweiten Dorsalstreifens) ohne Porenpunkte. Wangen stumpfwinklig vortretend. Flügeldecken kaum doppelt solang als zusammen breit, die Streifen am Grunde glatt. Basis des Halsschildes in flachem Bogen ausgeschnitten.

eurytus Fisch.

1' Seiten des Halsschildes mit zähnchenartig vortretenden Vorderwinkeln.

$2^{\prime \prime}$ Wangen stumpfwinklig vortretend. Flügeldecken kaum doppelt so lang als zusammen breit, die Streifen am Grunde glatt. Seiten, des Halsschildes leicht gerundet. Basis sehr schwach stumpfwinklig ausgeschnitten.

salinus Dej.

a" Dritter Zwischenraum der Flügeldecken weit von der Spitze mit einem Porenpunkte, der bisweilen einseitig fehlen kann. Außenseite der Hintertibien glatt, glänzend, selten fein quer gerunzelt. Die gekielte Mittellinie des Kinns reicht bis zur Basis und trennt die vor letzterer befindliche tiefe Grube genau in zwei Hälften. $23-32 \mathrm{~mm}$.

Stammform.

a' Dritter Zwischenraum ohne Porenpunkt. Außenseite der Hintertibien dicht fein punktuliert, matt. Auf dem Kinn reicht die 
gekielte Mittellinie nur bis zur Grube, diese nicht geteilt. $28 \mathrm{~mm}$. (Kreis Aresch. Gouv. Elisabethpol, 30. Mai 1902).

Reitteri m. nov.

2' Augenlappen stark eckig vorspringend, die Seiten des Halsschildes von den Vorderwinkeln bis zum Seitenzahne (Hinterwinkel) ganz gerade nach hinten konvergierend. Flügeldecken cylindrisch, lang, viel mehr wie doppelt so lang als zusammen breit. Die Streifen am Grunde sehr deutlich, die seitlichen sehr stark punktiert. Körper lang, cylindrisch. (ex. Reitter). cylindronotus Fald.

Eucarterus sparsutus Reitt. D. E. Z. 1898, p. 338 aus Kurdistan (Mardin) beschrieben, kommt auch im Kaukasus vor. Ich fing diese Art im Kreise Aresch, Gouv. Elisabethpol.

Cartodere costulata Reitt. Bisher aus Japan und Deutschland bekannt kommt auch im Kaukasus vor. Ich besitze diese Art aus Temir-Chan-Schura (1890 Tschitschérine) und Tiflis (25. Sept. 1902).

Ochodaeus Alleonis Fairm. Von dieser interessanten und fast unbekannten Art fing ich am 1. Mai 1902 ein $q$ im Kreise Aresch, Gouv. Elisabethpol. Ich war zuerst nicht sicher, ob es diese Art sei, weil mein Exemplar eine schwarzbraune Fühlerkeule besitzt, sonst paßte aber die Beschreibung, besonders das lange, schmale und spitze Schildchen, ganz gut. Später erhielt ich von Dr. Maljushenko ein Pärchen zur Bestimmung, das bei Elisabethpol gefangen wurde. Beide Exemplare besassen eine gelbe Fühlerkeule. Der Körper ist kürzer und gedrungener als bei $O$. chrysomeloides Schrank und O. integriceps Sem. Die Vorderschienen sind zweizähnig. Der Clypeus ist vorne stumpf gerundet mit einem sehr kleinen Höckerchen vorne in der Mitte, vor den Augen mit einer scharfen Querfurche. Die Flügeldecken sind flacher als bei den verglichenen Arten, die Streifen deutlich, ohne große Punkte. Die Zwischenräume sind schwach gewölbt, länger und dreireihig behaart. Die Hinterschenkel beim $q$ schwach gezähnt. Bei dem $\sigma^{x}$ sind die Vorder- und Mittelschenkel gezähnt, die Hinterschenkel mit sehr großem Zahn. Die Vorderschenkel haben auf der Vorderkante hinter der Mitte einen stumpfen kegelförmigen Zahn. L.: o $6 \mathrm{~mm}$.; † 4.5-5 mm. 


\section{$2 \mathrm{BHL}$ Biodiversity Heritage Library}

Koenig, Eugen Gregor. 1904. "Zweiter Beitrag zur Coleopteren-Fauna des Kaukasus." Wiener entomologische Zeitung 23, 140-142.

https://doi.org/10.5962/bhl.part.27197.

View This Item Online: https://www.biodiversitylibrary.org/item/44102

DOI: https://doi.org/10.5962/bhl.part.27197

Permalink: https://www.biodiversitylibrary.org/partpdf/27197

\section{Holding Institution}

Smithsonian Libraries

\section{Sponsored by}

Smithsonian

\section{Copyright \& Reuse}

Copyright Status: NOT_IN_COPYRIGHT

This document was created from content at the Biodiversity Heritage Library, the world's largest open access digital library for biodiversity literature and archives. Visit BHL at https://www.biodiversitylibrary.org. 